\title{
Investigation on the Microstructure and Mechanical Properties of Dissimilar Weld Joint for Inconel 617 Alloy and $12 \mathrm{Cr}$ Steel
}

\author{
Wang Peng ${ }^{1, *}$, Xu Huali ${ }^{2}$, Liu Xia ${ }^{1}$ \\ ${ }^{1}$ Shanghai Turbine Plant of Shanghai Electric Power Generation Equipment Co. Ltd., Shanghai, China \\ ${ }^{2}$ School of Materials Science and Engineering, Shanghai Jiaotong University, Shanghai, China
}

\section{Email address:}

wangpeng@shanghai-electric.com (Wang Peng)

${ }^{*}$ Corresponding author

\section{To cite this article:}

Wang Peng, Xu Huali, Liu Xia. Investigation on the Microstructure and Mechanical Properties of Dissimilar Weld Joint for Inconel 617 Alloy and 12Cr Steel. Science Discovery. Vol. 5, No. 1, 2017, pp. 53-58. doi: 10.11648/j.sd.20170501.19

Received: January 18, 2017; Accepted: February 18, 2017; Published: April 12, 2017

\begin{abstract}
The Inconel 617 nickel base alloy and 12Cr steel were welded by auto-TIG welding method, the microstructure and mechanical properties of dissimilar weld joint were investigated. The results showed that the optimized TIG welding process could obtain no defect dissimilar weld joint. Characterization on the microstructure of the weld joint showed that the weld metal was the coarsed columnar crystal with the secondary precipitation phase distribution in interdendritic which was growth with each other and through the weld layers. HAZ of $12 \mathrm{Cr}$ consisted of coarse grain zone, fine grain zone, and carbides near the fusion line of Inconel 617 change obviously. The hardness distribution of welded joint showed that HAZ near $12 \mathrm{Cr}$ steel existed tempered soften zone, the minimum hardness is $240 \mathrm{HV}$; hardness of weld was fluctuant, and the average hardness is about 330 HV because of the coarsed columnar crystal and the secondary precipitation phase distribution in interdendritic in weld; hardness of 617 side gradually reduced from the fusion line to the parent metal. Tensile test of the weld joint fractured at $\mathrm{HAZ}$ near $12 \mathrm{Cr}$; the lowest value of impact energy test occurred at HAZ near 617. Mechanical properties test result showed that the performance can satisfy the requirements.
\end{abstract}

Keywords: Inconel 617/12Cr Dissimilar Metal, Weld Joint, Microstructure, Mechanical

\section{Incone1 617 镍基合金与 $12 \mathrm{Cr}$ 钢异种材料 焊接接头组织与力学性能研究}

王朋 ${ }^{1 *}$, 徐华力 ${ }^{2}$, 刘霞 ${ }^{1}$

${ }^{1}$ 上海电气电站设备有限公司汽轮机厂, 上海, 中国

${ }^{2}$ 材料科学与工程学院, 上海交通大学, 上海, 中国

邮箱

wangpeng@shanghai-electric.com (王朋)

摘要：采用自动铇极氩弧焊（TIG）的方法完成了Incone1 617镍基合金与 $12 \mathrm{Cr}$ 钢异种材料焊接工艺试验, 针对焊接接 头的微观组织及力学性能进行表征与研究。研究结果表明: 采用TIG焊接方法及优化工艺可以获得无缺陷的异种材料焊 接接头。接头组织表征发现: 焊缝组织为粗大的柱状晶奥氏体组织, 二次析出相分布于枝晶间, 柱状晶在层间为联生 生长, 并穿过多层; 12Cr侧热影响区（HAZ）主要包括粗晶及细晶马氏体组织, 而617侧HAZ近熔合线附近碳化物变化明 
显。焊接接头硬度测试表明： $12 \mathrm{Cr}$ 侧HAZ存在硬度最低值为 $240 \mathrm{HV}$, 该处为回火软化区; 焊缝硬度波动较大, 平均硬度 约为 $330 \mathrm{HV}$, 这与焊缝中粗大的柱状晶组织及枝晶间的二次析出相有关; 617侧硬度由HAZ向母材逐渐降低。焊接接头的 拉伸断裂发生在12Cr侧HAZ硬度最低值处, 617侧HAZ冲击功最低, 接头的力学测试结果表明性能均满足使用要求。

关键词: 617/12Cr异种材料, 焊接接头, 组织表征, 力学性能

\section{1. 引言}

近年来, 能源与环境问题成为影响人类生活的主要问 题, 火力发电将长期处于我国的能源主要地位, 因此, 要 努力解决碳排放并提高发电效率，通过提高蒸汽温度，提 高热效率并降低 $\mathrm{CO}_{2}$ 排放为当前首选的解决方案 $[1]$ 。当蒸 汽温度提高到 $700^{\circ} \mathrm{C}$ 以上, 耐热钢已经不能满足使用需求, 镍基合金由于其具有优良的高温性能和组织稳定性, 成为 高温用材料的最佳选择 [2-4]。

Inconel 617 镍基合金是固溶强化型的 $\mathrm{Ni}-\mathrm{Cr}-\mathrm{Co}-\mathrm{Mo}$ 高温合金, 该合金由于其较好的高温力学性能和抗蠕变性 能, 广泛地应用在电站、航天、化工以及核工业, 因此成 为电站用 $700^{\circ} \mathrm{C}$ 超超临界机组的首选材料之一 $[5]$ 。由于镍 基合金价格相对较高, 且不同构件工作温度不同, 可以在 温度较低、应用风险较小的区域选择相对便宜的材料, 充 分发挥各种材料的潜能, 异种材料连接成为构件制造的有 效途径。镍基合金与合金钢异种材料焊接时，由于两种材 料的化学成分、组织及力学性能差异较大, 会给焊接接头 带来高组织梯度、大应力梯度等难题。因此, 有必要针对 异种材料焊接材料选择、焊接工艺规范、焊后热处理、接 头组织以及力学性能等方面开展研究, 从而推动异种材料
焊接接头在构件制造中的应用。Hosseini H S 等 [6] 人研 究了 617 和 310 异种材料焊接时, 选择了三种焊接材料, 研 究结果表明, 617焊接材料室温下表现出更加理想的焊接 接头综合性能。Ahmad H等 [7] 人研究了 617 和 $12 \mathrm{Cr}$ 异种材 料焊接接头的力学性能和腐蚀性能, 初步得到了室温下焊 接接头的拉伸性能, 断裂位置均在 $12 \mathrm{Cr}$ 侧附近。

本文采用自动铇极氩弧焊 (TIG) 开展Inconel 617 镍基合金与 $12 \mathrm{Cr}$ 钢试板的对接试验, 研究焊接工艺参数及 热处理规范对接头质量的影响, 优化焊接工艺参数。同时, 针对异种材料焊接接头的组织及接头力学性能进行研究, 获得Inconel 617 镍基合金与12Cr 异种材料焊接接头的组 织变化规律及力学性能, 为 617 与 $12 \mathrm{Cr}$ 异种材料焊接接头 在行业中的应用提供理论及数据支撑。

\section{2. 试验材料和方法}

试验使用的材料是Incone1 617 镍基合金试板以及 12Cr 钢试板, 其中Inconel 617镍基合金供货态为固溶热 处理状态。Inconel 617镍基合金及12Cr钢的主要的化学 成分见表1所示。

表1 Incone1 617镍基合金及12Cr钢的主要化学成分（Wt.\%）。

\begin{tabular}{llllllllllll}
\hline & $\mathrm{C}$ & $\mathrm{Si}$ & $\mathrm{Mn}$ & $\mathrm{P}$ & $\mathrm{S}$ & $\mathrm{Cr}$ & Mo & Ni & $\mathrm{Al}$ & $\mathrm{V}$ & $\mathrm{Co}$ \\
\hline Incone1 & 617 & 0.052 & 0.062 & 0.031 & 0.008 & 0.006 & 21.63 & 9.15 & Bal. & 1.02 & - \\
12Cr钢 & 0.13 & 0.08 & 0.40 & 0.006 & 0.001 & 10.57 & 1.02 & 0.78 & 0.004 & 0.18 & - \\
\hline
\end{tabular}

两块试板尺寸为 $400 \times 130 \times 50 \mathrm{~mm}$, 采用刚性拘束固定 组对, 坡口间隙 $13 \mathrm{~mm}$ 。采用自动铇极氩弧焊 $(\mathrm{TIG})$ 的方 法多层单道焊接而成。焊接完成后, 进行 $680^{\circ} \mathrm{C} \times 10 \mathrm{~h}$ 的焊 后热处理, 然后进行取样, 对接头组织和力学性能进行测 试分析。

\section{3. 结果与讨论}

\section{1. 焊接接头组织分析}

焊接接头的力学性能与其微观组织有着密切的联系, 因此首先针对该异种接头的微观组织进行分析。图1 所示 为焊接接头的宏观形貌, 其中焊接接头包括了母材 (BM), 热影响区 (HAZ), 以及焊缝金属 (WM) 三个部分。焊缝宽度 约为 $13 \mathrm{~mm}$, 焊缝组织呈现柱状晶形貌, 具有多层特征, 层与层之间柱状晶为联生生长, 并穿过多层生长。12Cr 钢侧HAZ在宏观上观察较为明显。从宏观形貌上可以看出, 焊接接头的各区域分布, 且无气孔、裂纹等缺陷存在, 表 明该焊接工艺是可行的。

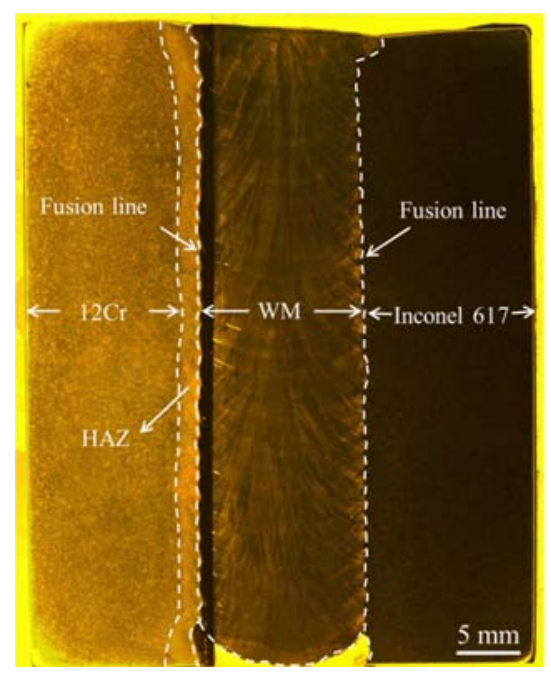

图1 焊接接头的宏观形貌。

图2所示为 $12 \mathrm{Cr}$ 钢母材的金相组织, $12 \mathrm{Cr}$ 钢母材的主 要组织为板条马氏体, 原始奥氏体晶粒较为粗大, 表明该 
组织具有较高的强度和硬度。图3 所示为Incone1 617 母 材的金相组织, 母材为奥氏体组织, 晶粒尺寸较大。基体 中存在大量的退火孪晶和 $\mathrm{M}_{23} \mathrm{C}_{6}$ 及 $\mathrm{Ti}(\mathrm{C}, \mathrm{N})$ 析出相 $[7-10]$ 。
其中 $\mathrm{M}_{23} \mathrm{C}_{6}$ 数量相对较多, 分布不均匀, 可观察到 $\mathrm{M}_{23} \mathrm{C}_{6}$ 碳化 物条带。 Ti $(\mathrm{C}, \mathrm{N})$ 数量较少, 颗粒较大, 在光学显微镜下 呈橘红色, 分布在晶界或晶内。
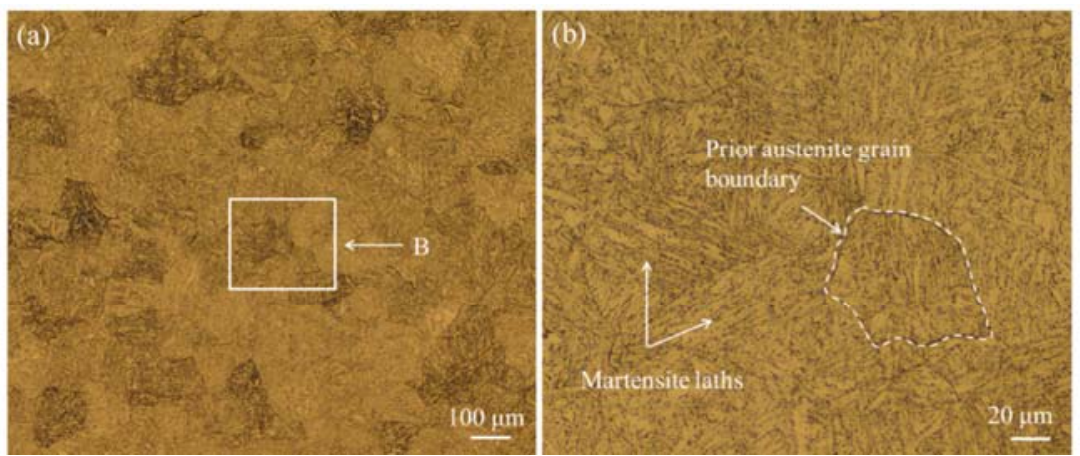

图2 12Cr钢母材组织: (a)母材组织 (b) 粗大的原奥氏体晶粒。
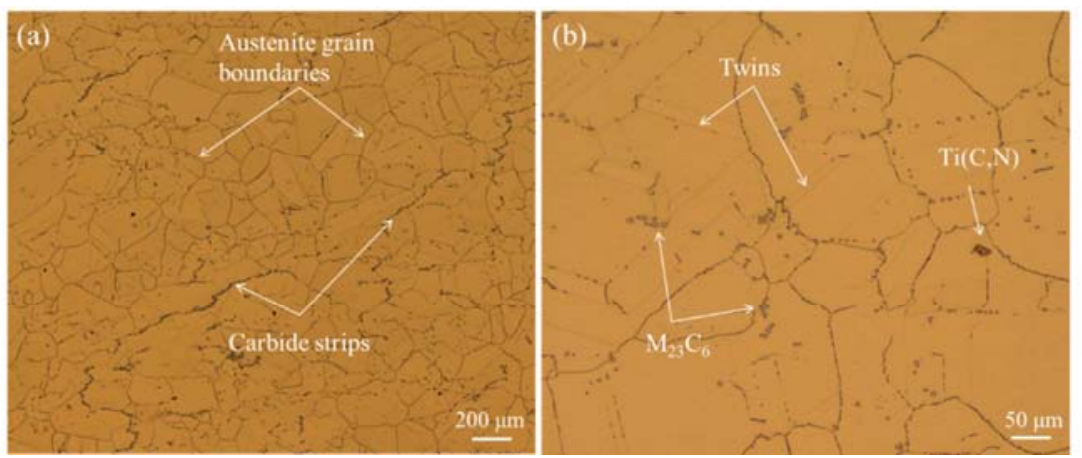

图3 Incone1 617母材显微组织:

(a) 母材组织, (b) 母材中析出相。

图4 所示为焊缝显微组织, 柱状晶逆着热传导方向生 长, 每一层焊道厚度约为 $1.5 \mathrm{~mm}$ 。层与层之间柱状晶为联 生生长。柱状晶由生长方向一致的枝晶构成, 由于 Cr、Mo、 $\mathrm{Ti}$ 等元素的偏析作用，枝晶间有大量的二次相生成。层间
与焊层中组织主要区别在于枝晶间二次析出相含量不同。 层间由于金属再熔, 二次相析出较少, 焊层由于下一层的 加热作用，枝晶间二次相析出较多。
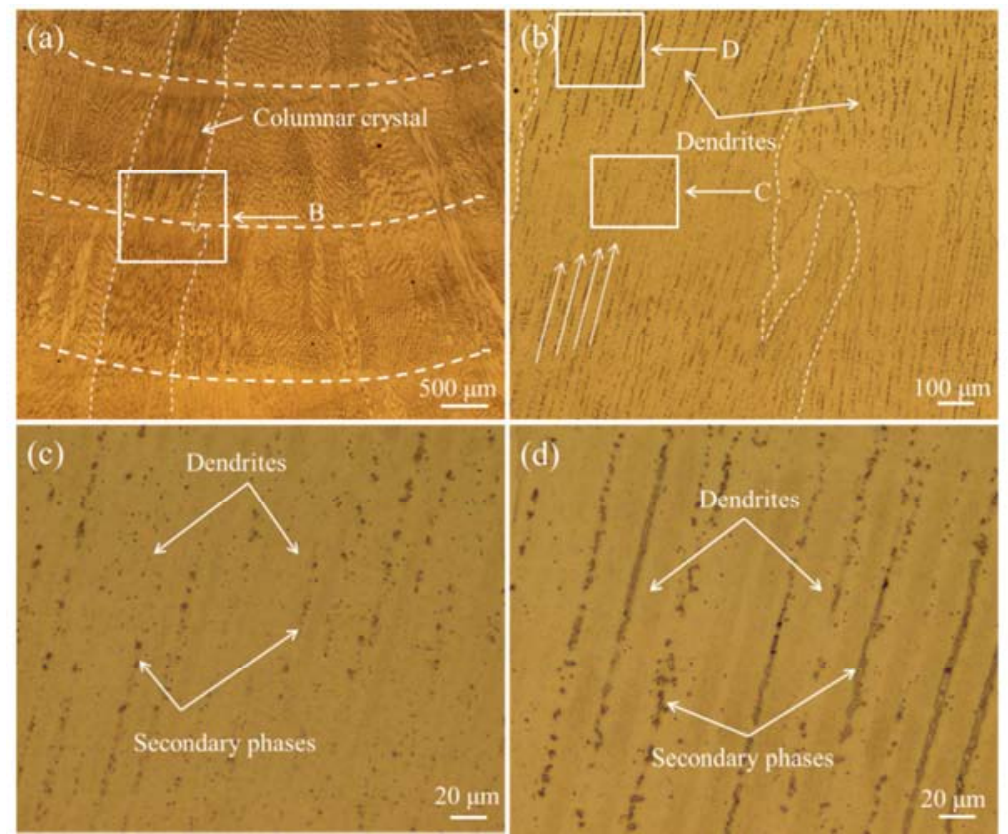

图4 焊缝显微组织: (a) 焊缝柱状晶组织, (b) 层间及周围组织, (c) 层间枝晶组织, 枝晶间二次相含量较少, (d) 焊层枝晶组织, 枝晶间二次相含 量较多。 
图5 所示为 $12 \mathrm{Cr}$ 钢侧热影响区组织, 主要为粒状回火 马氏体。由于焊接热循环的作用, HAZ 的不同区域的组织 呈现出不同的特征。图5 (b)-(d) 分别表征熔合线附近的粗 晶区组织、细晶区组织以及过回火区组织。焊接过程中,
粗晶区与细晶区的温度高于Ac3。在奥氏体化的过程中由 于临近熔合线区域过热, 晶粒生长粗大, 为粗晶区; 细晶 区由于焊接热循环温度相对较低, 晶粒细小。过回火区主 要为回火马氏体组织。
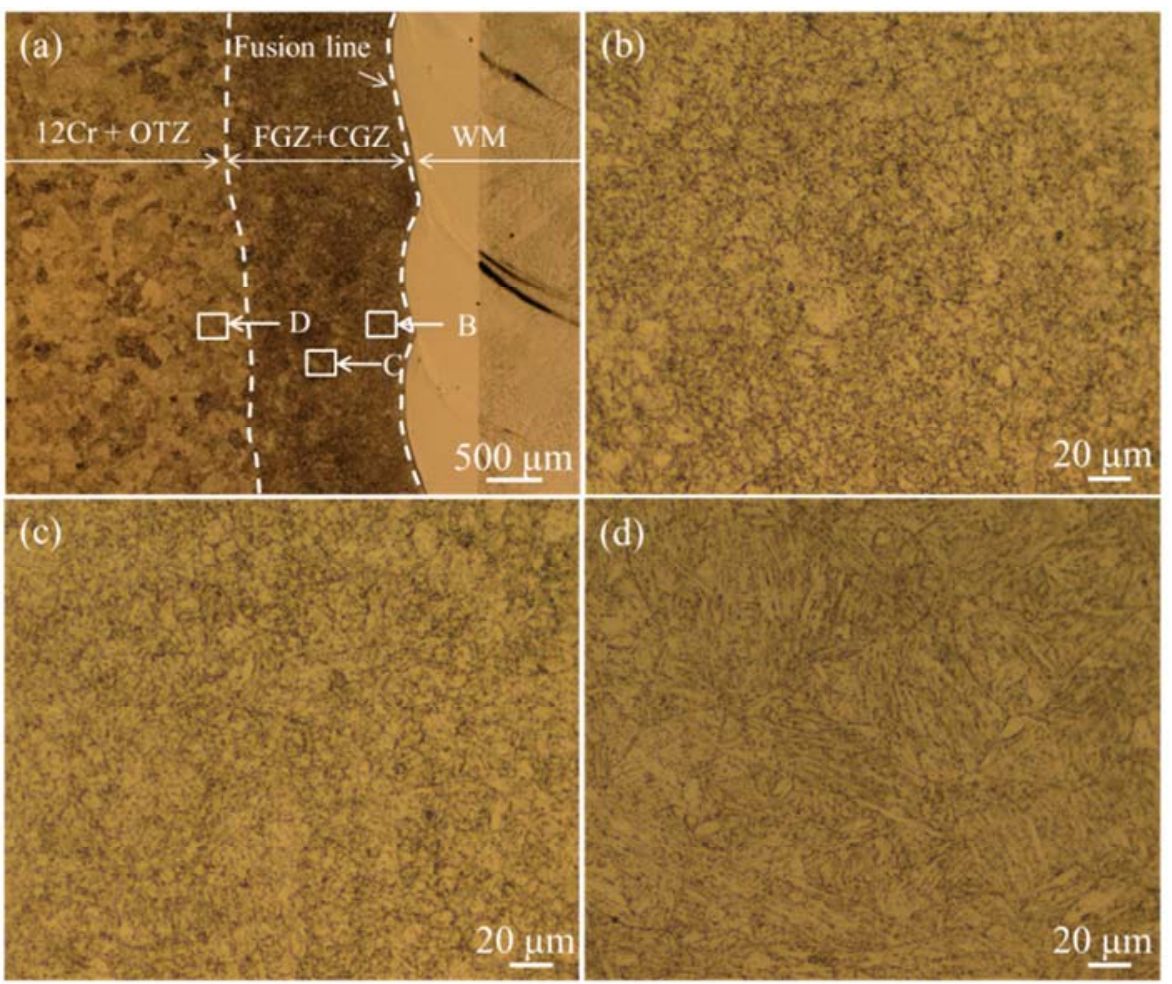

图5 12Cr侧热影响区显微组织: (a) 12Cr侧热影响组织, (b) 粗晶区组织, (c) 细晶区组织, (d) 过回火组织。

图6 所示为Incone1 617侧热影响区附近显微组织, 其主要组织仍为奥氏体, 晶粒大小没有明显的变化, 组织上很 难区分热影响区的宽度。在距熔合线 $100 \mu \mathrm{m}$ 范围内, 原始碳化物在焊接热的作用下与基体发生反应, 形态发生明显变 化。

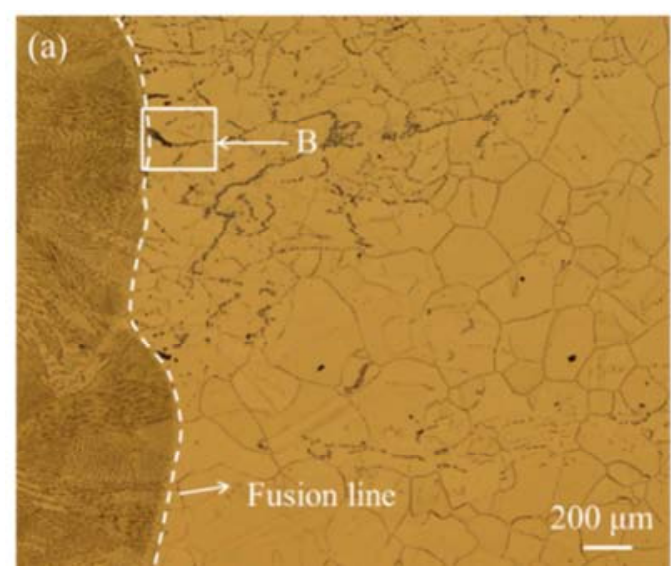

图6 Incone1 617侧热影响区附近显微组织:

\section{2. 焊接接头的硬度分布}

焊接接头的硬度分布与各区域的组织形态及力学性 能相关。因此针对全焊接接头沿其长度方向进行硬度测试, 采用9. $8 \mathrm{~N}$ 的载荷, $0.25 \mathrm{~mm}$ 间隙, 持续时间为 $15 \mathrm{~s}$ 。硬度测 试的结果如图7 所示。焊缝中硬度波动较大，在330HV左

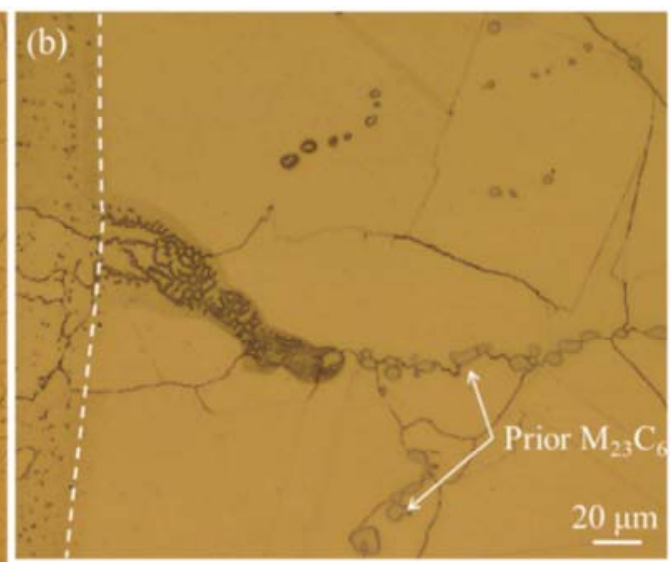

(a) 母材靠近熔合线附近组织, (b) 局部放大。

右波动, 最低达到 $300 \mathrm{HV}$, 最高达到 $350 \mathrm{HV}$ 。这与焊缝中 粗大的柱状晶组织及枝晶间的二次相有关。

在 $12 \mathrm{Cr}$ 钢侧, 硬度最高点出现在 $12 \mathrm{Cr}$ 侧热影响区的粗 晶区 (CGZ) , 随着远离熔合线, 硬度值逐渐下降, 至过 回火区 (OTZ) 处下降最低至 $240 \mathrm{HV}$ 。在过回火区中硬度 有所回升, 并在 $12 \mathrm{Cr}$ 母材 (BM) 处达到稳定, 稳定在 $270 \mathrm{HV}$ 。 
由此可以推测出, $12 \mathrm{Cr}$ 侧热影响区的宽度约为 $3 \mathrm{~mm}$, 其中 CGZ 与FGZ的宽度约为 $1.5 \mathrm{~mm}$, OTZ的宽度约为 $1.5 \mathrm{~mm}$ 。在 Incone1 617侧, 硬度最高出现在靠近熔合线处, 随着远 离熔合线, 硬度值逐渐下降, 距熔合线 $10 \mathrm{~mm}$ 达到稳定, 稳 定在 $240 \mathrm{HV}$ 范围, 但总体波动较大, 这与母材中较大的奥 氏体晶粒尺寸及分布不均匀的碳化物有关。

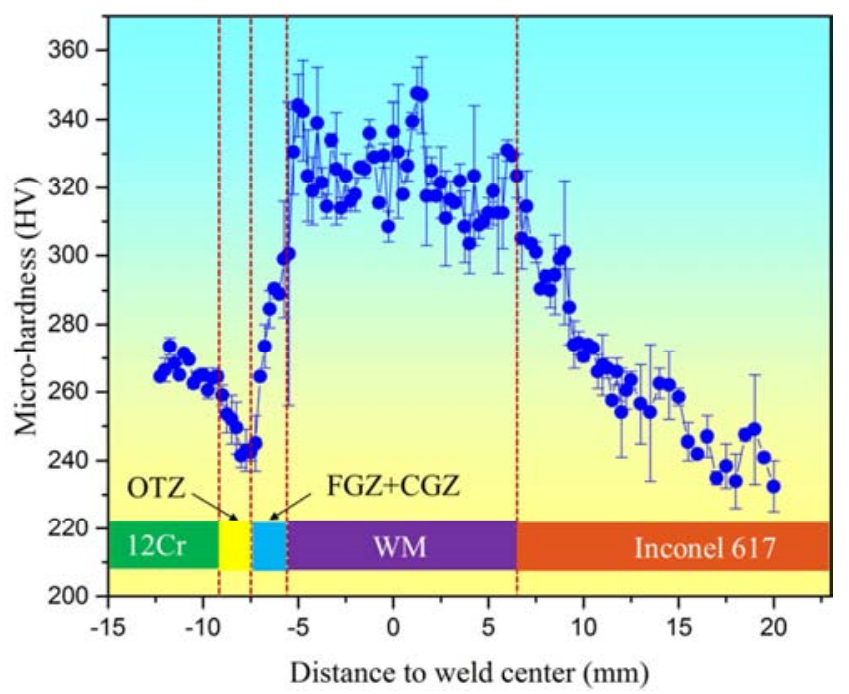

图7 全焊接接头硬度分布图。

\section{3. 焊接接头的拉伸性能}

采用电火花线切割的方法取焊后热处理后的焊接接 头试料, 并车制拉伸试样, 原始标距取为 $25 \mathrm{~mm}$, 覆盖焊缝 及两侧的热影响区。测试焊接接头的室温 $\left(25^{\circ} \mathrm{C}\right) 、 550{ }^{\circ} \mathrm{C}$ 及 $600^{\circ} \mathrm{C}$ 下的拉伸性能, 所有拉伸试样均断在 $12 \mathrm{Cr}$ 钢侧 $\mathrm{HAZ}$ 的过回火区, 拉伸试样断裂照片如图8所示, 试验结果如 图9所示。室温下, Inconel 617/12Cr焊接接头塑性变形 明显, 而 $550{ }^{\circ} \mathrm{C}$ 及 $600{ }^{\circ} \mathrm{C}$ 下, 塑性变形明显降低, 表明 Incone1 617母材具有较好的热强性。从图8可以看出, 随 着温度的升高, 抗拉强度和屈服强度不断减小, 屈强比增 大。
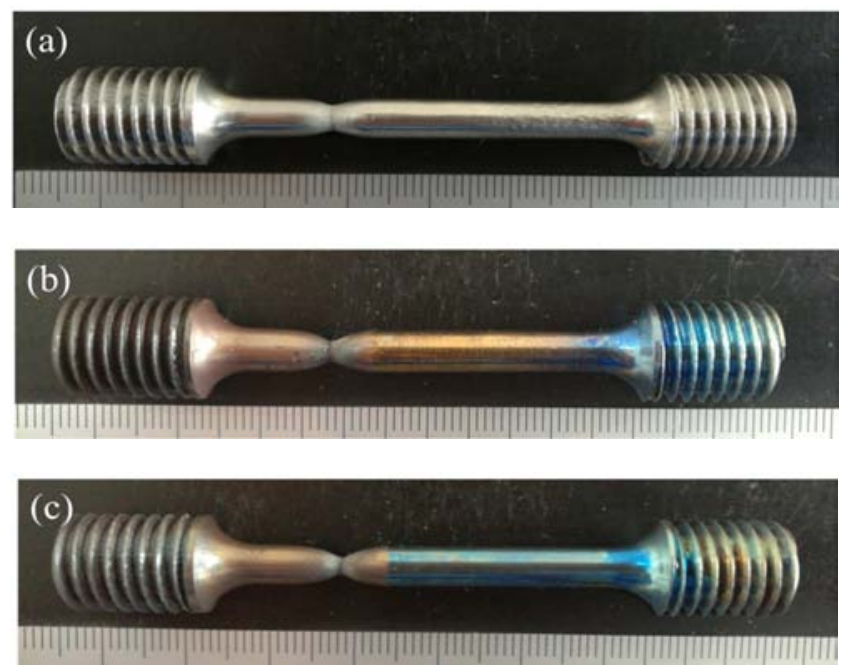

图8 不同温度焊接接头拉伸试样断裂照片: (a) $25^{\circ} \mathrm{C}$, (b) $550{ }^{\circ} \mathrm{C}$, (c) $600^{\circ} \mathrm{C}$ 。

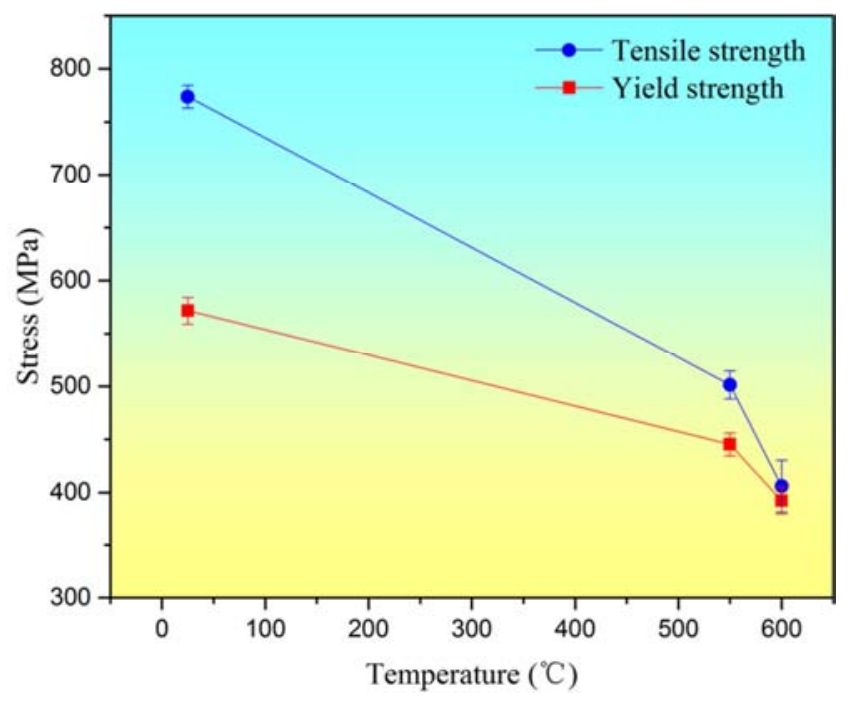

图9 焊接接头的拉伸性能。

\section{4. 焊接接头的冲击性能}

采用电火花线切割的方法取焊后热处理后的焊缝及 两侧母材的冲击试料, 并加工冲击试样。V 型缺口中心分 别在Incone1 617母材、Incone1 617侧HAZ、焊缝中心、 $12 \mathrm{Cr}$ 侧HAZ以及 $12 \mathrm{Cr}$ 钢母材处。对各个区域的室温冲击进 行测试, 冲击试验结果如图10所示, 可以看出, 焊接接头 各区域冲击韧性较好, 均高于规范要求值。

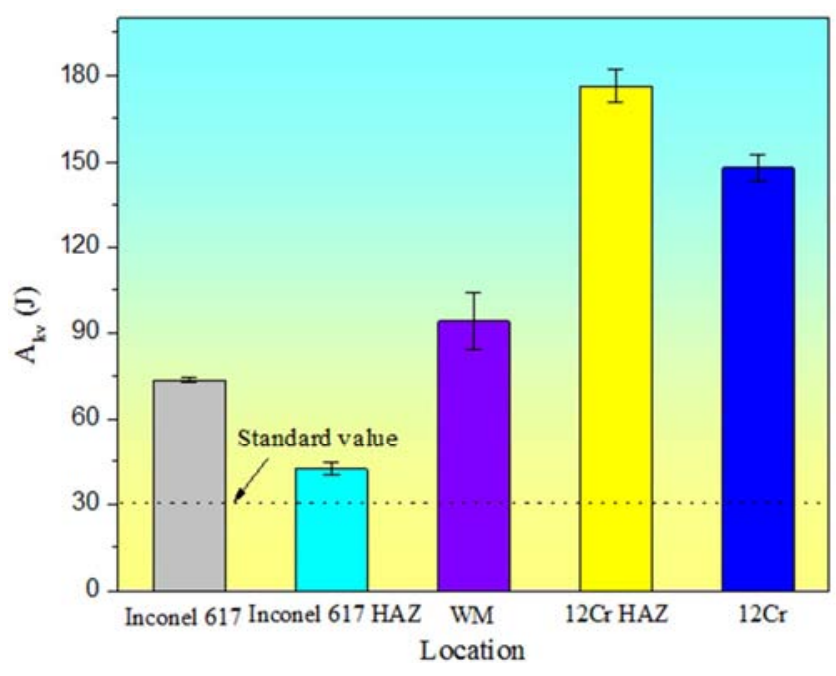

图10 焊接接头各区域的冲击性能。

\section{4. 结论}

本文研究了采用钨极氩弧焊方法获得的Incone1 617 镍基合金与 $12 \mathrm{Cr}$ 钢焊接接头, 并针对该异种材料焊接接头 的组织及接头力学性能进行研究。研究结果表明, 采用 TIG 焊接方法及优化工艺可以获得无缺陷的异种材料焊接接 头; 获得的焊缝组织为粗大的柱状晶奥氏体组织, 二次析 出相分布于枝晶间, 柱状晶在层间为联生生长, 并穿过多 层； $12 \mathrm{Cr}$ 侧热影响区（HAZ）主要包括粗晶及细晶马氏体 组织, 而617侧HAZ近熔合线附近碳化物变化明显; 接头硬 度显示, 焊缝硬度波动较大, 平均硬度约为 $330 \mathrm{HV}$, 这与 
焊缝中粗大的柱状晶组织及枝晶间的二次析出相有关; 焊 接接头的拉伸断裂发生在 $12 \mathrm{Cr}$ 侧HAZ硬度最低值处, 617 侧HAZ冲击功最低, 接头的力学测试结果表明性能均满足 使用要求。

\section{参考文献}

[1] 田仲良, 包汉生, 何西扣, 等. $700^{\circ} \mathrm{C}$ 汽轮机转子用耐热合 金的研究进展 $[J]$. 钢铁, 2015，27(2):54-60.

[2] Bohan, WANG, Shufang. Aging Precipitation Behavior and Mechanical Properties of Inconel 617 Superalloy[J]. Acta Metallurgica Sinica, 2013, $26(3): 307-312$.

[3] 王会阳, 安云岐, 李承宇, 等. 镍基高温合金材料的研究 进展 [J]. 材料导报, 2011 (S2) : 482-486.

［4］江河，董建新，张麦仓, 等. $700^{\circ} \mathrm{C}$ 超超临界锅炉管用 $617 \mathrm{~B}$ 合金时效组织演变 [J]. 稀有金属材料与工程, 2016 (4) : 982-989.
[5] Mankins WL, Hosier JC, Bassford TH. Microstructure and phase stability of Inconel alloy 617. Metall Trans $1974 ; 5: 2579-90$.

[6] Hosseini H S, Shamanian M, Kermanpur A. Characterization of microstructures and mechanical properties of Inconel $617 / 310$ stainless steel dissimilar welds $[J]$. Materials Characterization, 2011, $62(4): 425-431$.

[7] Ahmad H, Hwang J, Ju L, et al. An Assessment of the Mechanical Properties and Microstructural Analysis of Dissimilar Material Welded Joint between Alloy 617 and 12Cr Steel[J]. 2016, 6(10).

［8］杨仁杰，刘文，芦凤桂. Incone1 617镍基合金焊接接头热 影响区组织和性能分析 [J]. 上海金属, 2015, 37 (4) :26-29.

[9］郭岩, 周荣灿, 张周博, 等. 镍基合金 $617 \mathrm{~B}$ 的组织结构与 力学性能稳定性 [C]// 中国电机工程学会年会. 2014 .

[10] 王硕, フ旺战, 杨权, 等. $700^{\circ} \mathrm{C}$ 关键部件验证试验平台用 $617 \mathrm{~B}$ 合金焊接接头组织和力学性能研究 $[\mathrm{J}]$. 动力工程学 报, 2016, 36(10):849-852. 